\title{
Analysis of petroleum-contaminated soils by diffuse reflectance spectroscopy and sequential ultrasonic solvent extraction-gas chromatography
}

Reuben N. Okparanma

Frederic Coulon

Abdul M. Mouazen*

a.mouazen@cranfield.ac.uk

mouazen2002@yahoo.com

Department of Environmental Science and Technology, Cranfield University, Cranfield, MK43 0AL Bedfordshire, United Kingdom

${ }^{*}$ Corresponding author.

\section{Abstract}

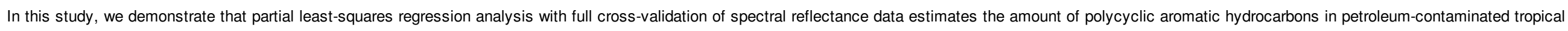

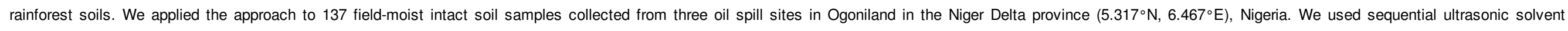

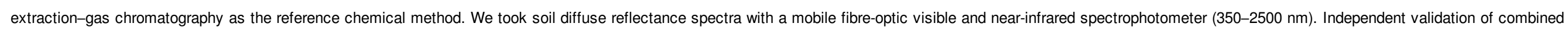

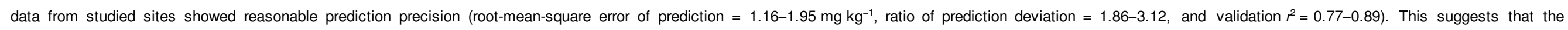
methodology may be useful for rapid assessment of the spatial variability of polycyclic aromatic hydrocarbons in petroleum-contaminated soils in the Niger Delta to inform risk assessment and remediation.

This approach may be used to collect large spatial data at reduced cost and time to assess the variability of polycyclic aromatic hydrocarbons in petroleum release sites.

Keywords: Chemometrics; Near-infrared spectroscopy; Hydrocarbon; Soil

\section{Introduction}

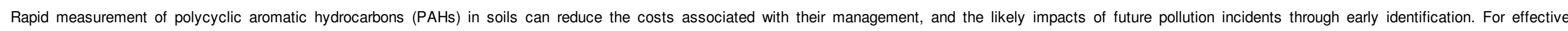

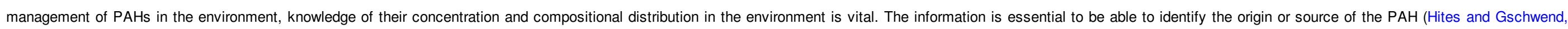

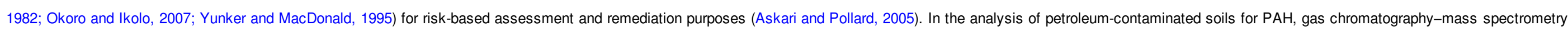

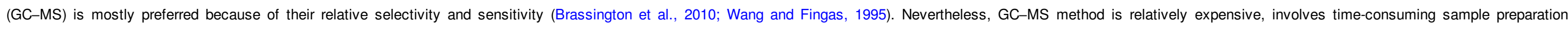

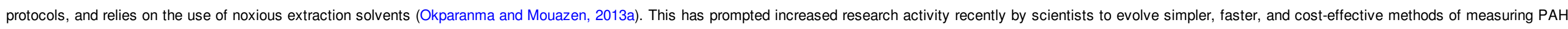
in contaminated soils to complement the conventional methods.

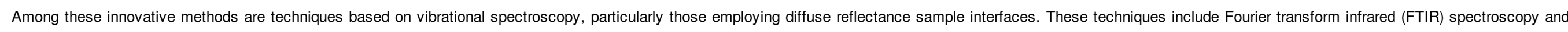

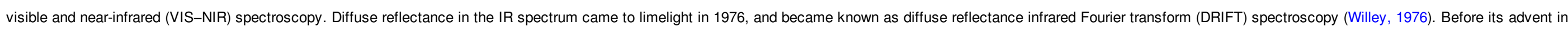

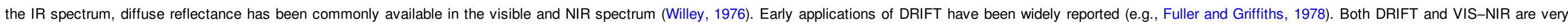

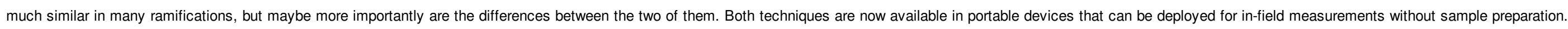

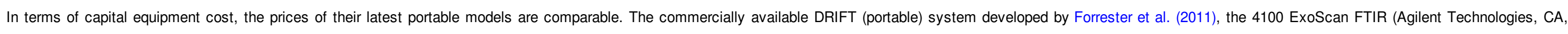

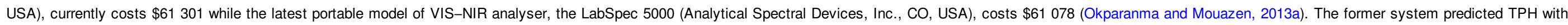




\section{ELSEVIER_ENPO_7234}

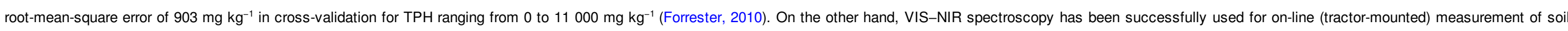

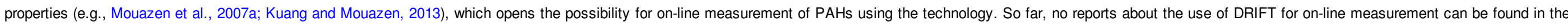
literature.

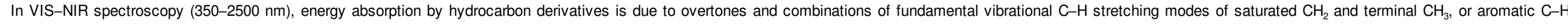

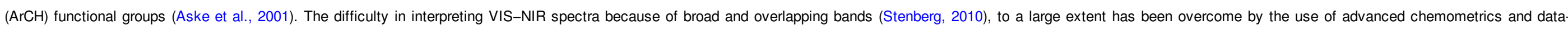

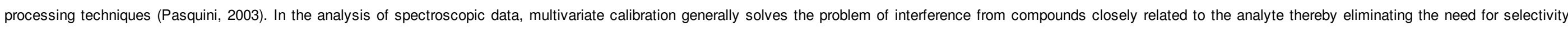

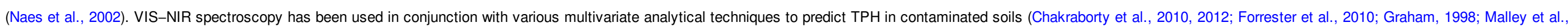

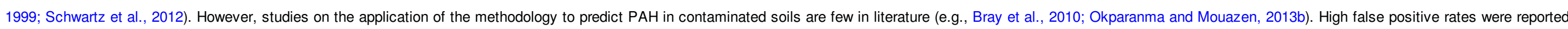

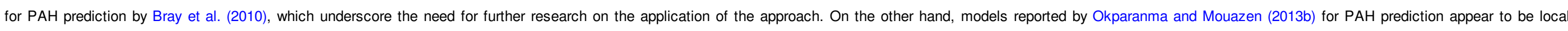

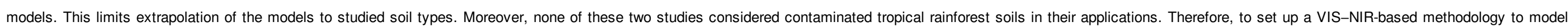
VIS-NIR spectra for broader environmental application, it is essential to expand the approach to cover a wider range of soil types and environmental conditions.

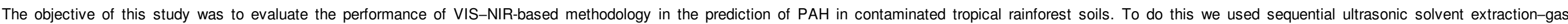

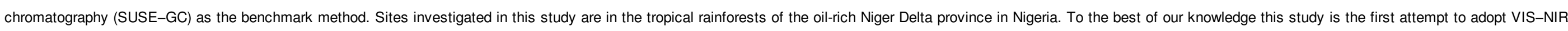
spectroscopy for the determination of hydrocarbons in contaminated arable lands in Nigeria.

\section{Materials and methods}

\subsection{Brief description of the study area}

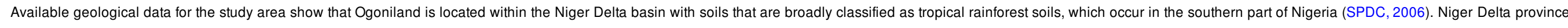

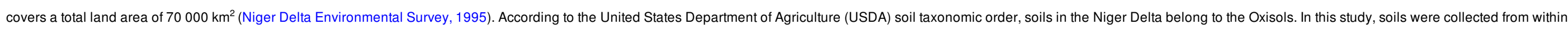

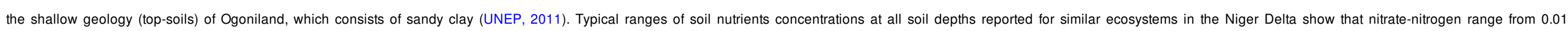

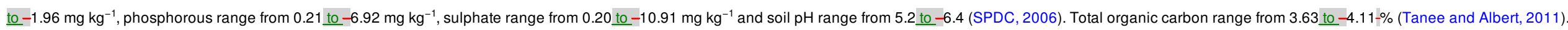

\subsection{Sample collection}

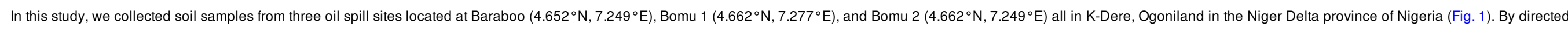

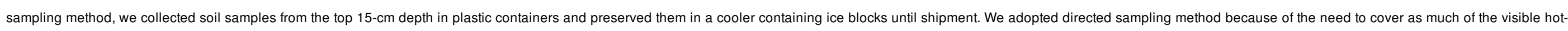

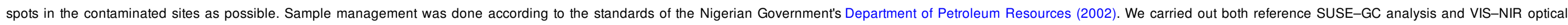
scanning of soil samples in the Environmental Analytical Facility of Cranfield University, United Kingdom.
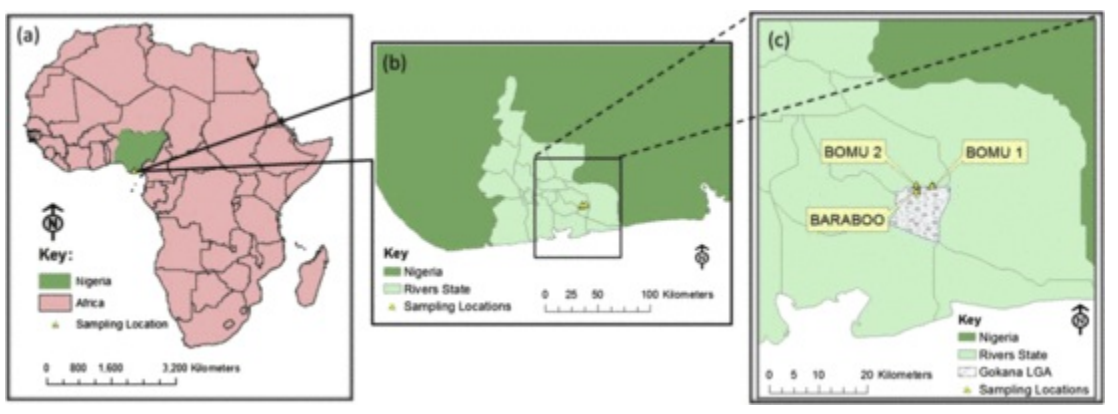

Online view $\square$ Print view 


\section{ELSEVIER_ENPO_7234}

Fig. 1 Sampling locations: (a) Nigeria, (b) Rivers State in the Niger Delta province, and (c) Gokana Local Government Authority in Ogoniland (Datum and Projection: GCS WGS 1984. Shape files source: ESR ${ }^{\circledR}$, CA, USA).

\subsection{Reference chemical analysis of PAHs}

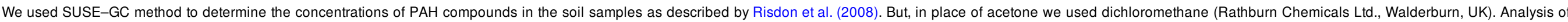

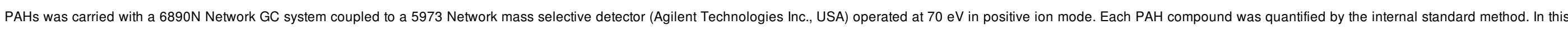

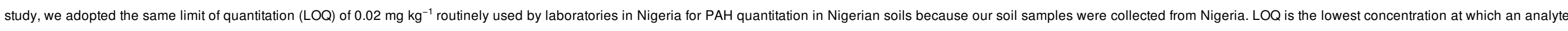

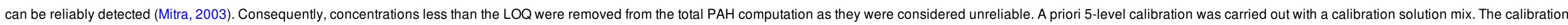

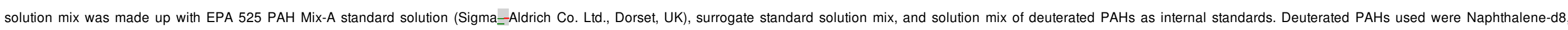

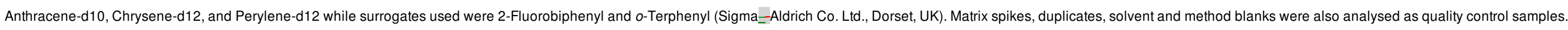

\subsection{Accuracy, precision, and experimental uncertainty of reference PAH analytical method}

Accuracy of the reference SUSE-GC chemical method was evaluated from the percent recovery of the surrogate spiked into the sample prior to extraction according to equation (1).

\section{$\%$ Recovery $=100 \times \frac{\text { Measured concentration }}{\text { Theoretical concentration }}$}

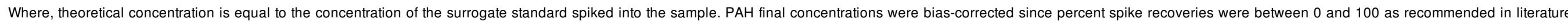

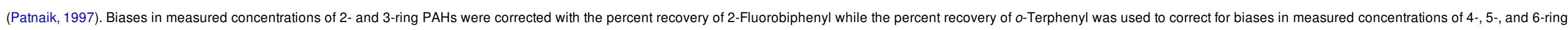

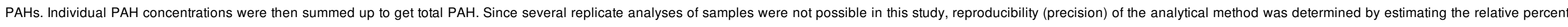
difference of duplicate analyses of one sample in each batch of samples as recommended in literature (Patnaik, 1997). Relative percent difference was deduced by means of equation (2).

Relative percent difference $(\%)=\frac{\left(a_{1}-a_{2}\right)}{\frac{\left(a_{1}+a_{2}\right)}{2}} \times 100$

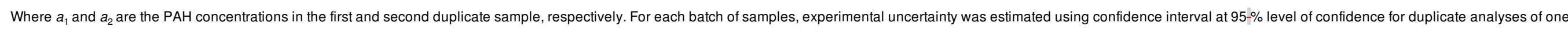
randomly chosen sample. The confidence interval was deduced by the well-known expression in equation (3).

Confidence interval $=\bar{x} \pm \frac{t s}{\sqrt{n}}$

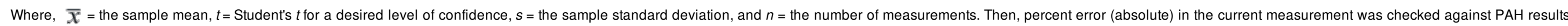

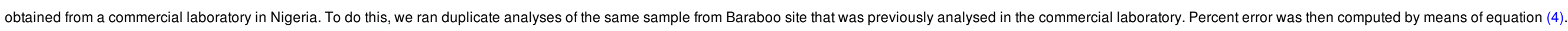

$\%$ Error $=\left|\frac{T-E}{T}\right| \times 100$

Where, $T=$ "known" PAH value from commercial laboratory, and $E=$ measured mean PAH value from current study.

\subsection{Optical measurement of soil samples}

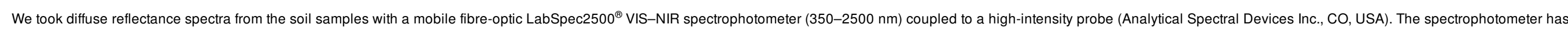

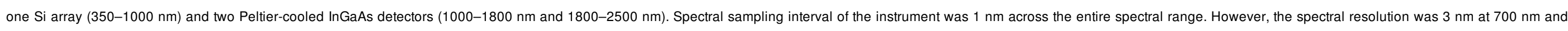

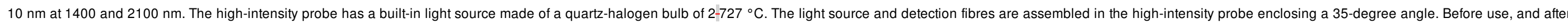

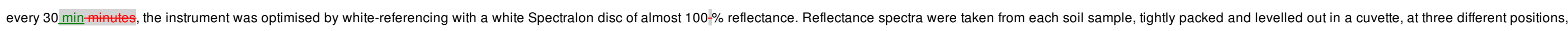




\section{ELSEVIER_ENPO_7234}

$120^{\circ}$ apart. Each sample was scanned three times at each position and averaged before spectral pre-processing and multivariate analysis.

\subsection{Spectral pre-processing}

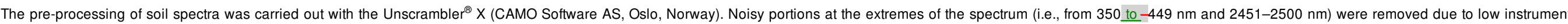

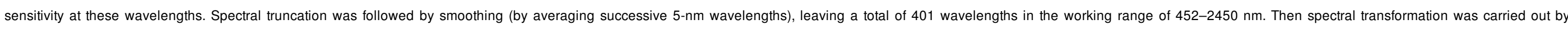

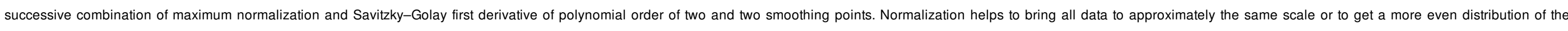

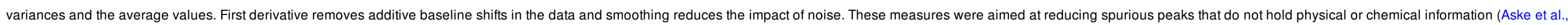
2001; Naes et al., 2002)

\subsection{Partial least-squares (PLS) regression analysis}

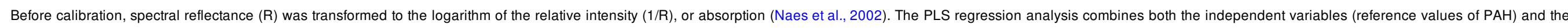

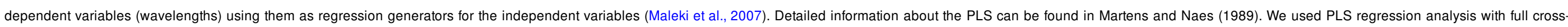

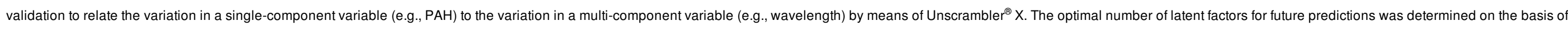

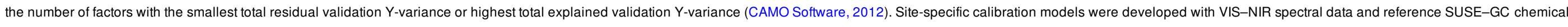

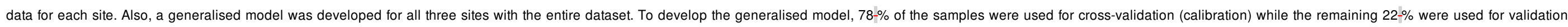
(prediction). The ratio of calibration/validation samples was chosen to ensure an equal representation of samples in the validation set by randomly choosing ten samples from each site.

\subsection{Statistical evaluation of model performance}

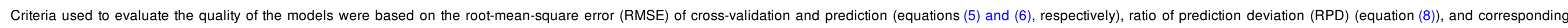

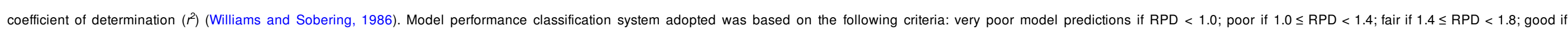
$1.8 \leq \mathrm{RPD}<2.0$; very good if $2.0 \leq \mathrm{RPD}<2.5$; and excellent if RPD $>2.5$ (Viscarra Rossel et al., 2006a).

$\mathrm{RMSECV}=\sqrt{\frac{\sum_{i=1}^{N}\left(\hat{\mathrm{y}}_{\mathrm{CV}_{i} i}-\mathrm{y}_{i}\right)^{2}}{N}}$

$\operatorname{RMSEP}=\sqrt{\frac{\sum_{i=1}^{N_{\mathrm{P}}}\left(\widehat{\mathrm{y}}-\mathrm{y}_{i}\right)^{2}}{N_{\mathrm{P}}}}$

$\mathrm{RMSE}=\sqrt{\mathrm{MSE}}=\sqrt{E(\hat{\mathrm{y}}-\mathrm{y})^{2}}$

$\mathrm{RPD}=\frac{\mathrm{SD}}{\mathrm{RMSE}}$

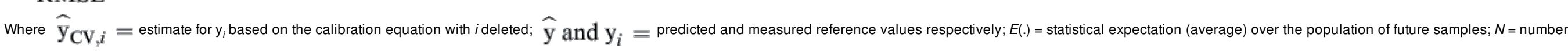
of samples in the set; and SD = standard deviation of the measured reference values.

\subsection{Outlier detection techniques}

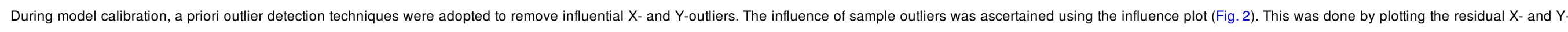

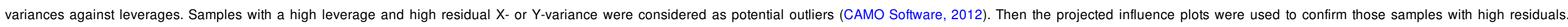

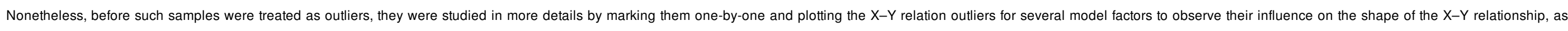




\section{ELSEVIER_ENPO_7234}

recommended in literature (CAMO Software, 2012).

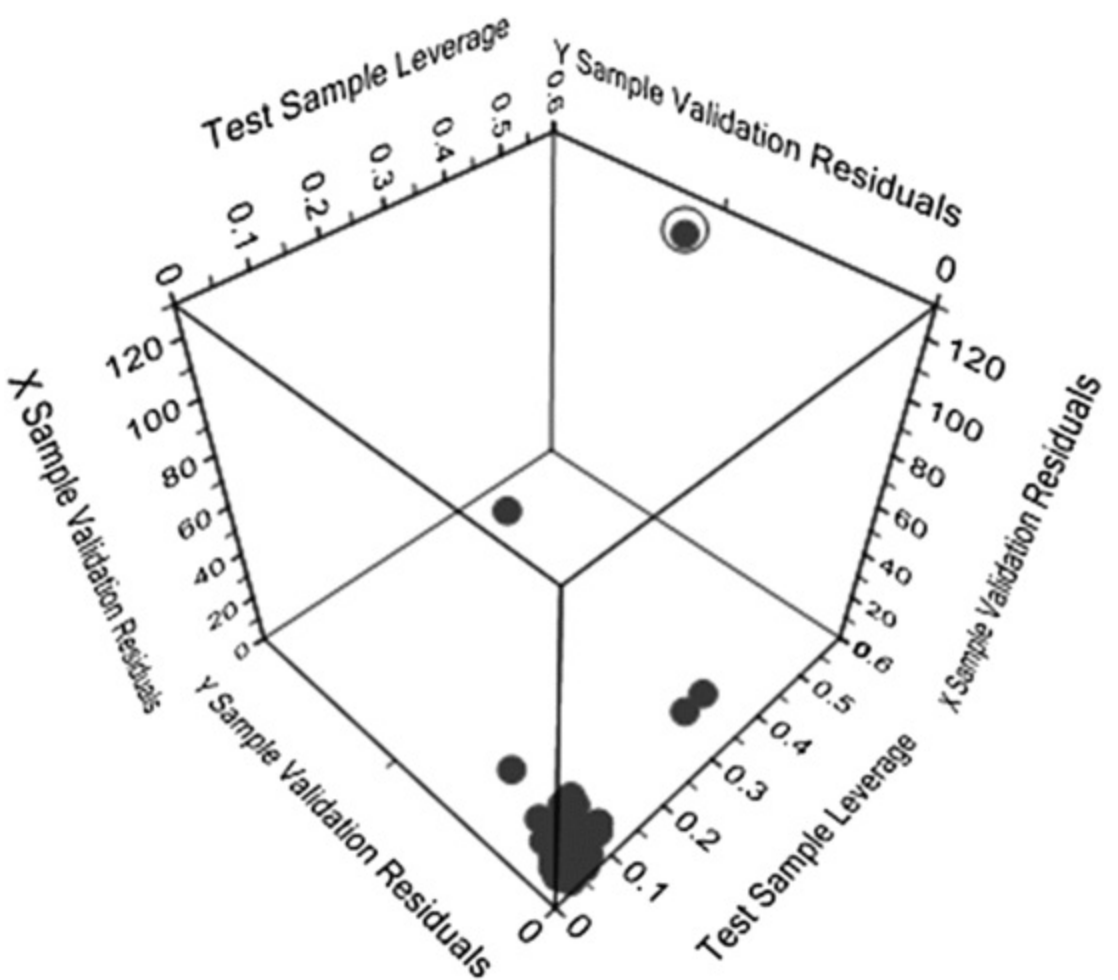

Fig. 2 Detection of outliers after partial least-squares regression analysis. A potential sample outlier, marked in circle, detected among samples from Baraboo site in Ogoniland, Niger Delta province of Nigeria is shown as an example.

\section{Results and discussion}

\subsection{Accuracy, precision and level of uncertainty in reference SUSE-GC analysis of PAH}

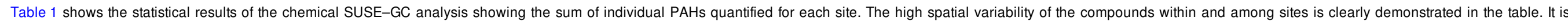

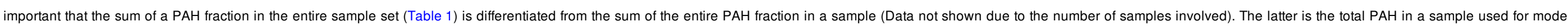

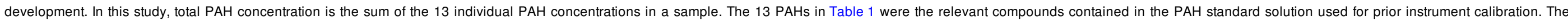

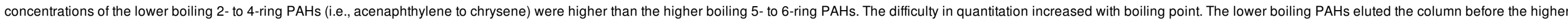

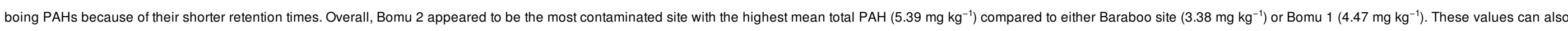
be confirmed from Table 1 by adding the respective mean values.

Table 1 Statistics of the chemical analysis result showing the sum of individual polycyclic aromatic hydrocarbons (PAHs) quantified for each site by reference sequential ultrasonic solvent extraction-gas chromatography (SUSE-GC).

PAH

LOQ $\left(\mathrm{mg} \mathrm{kg}^{-1}\right)$

Baraboo

Bomu 1

Bomu 2

$N \quad$ Min. $\left(\mathrm{mg} \mathrm{kg}^{-1}\right) \quad$ Max. $\left(\mathrm{mg} \mathrm{kg}^{-1}\right) \quad$ Mean $\left(\mathrm{mg} \mathrm{kg}^{-1}\right) \quad \begin{gathered}\text { Range } \\ \left(\mathrm{mg} \mathrm{kg}^{-1}\right)\end{gathered}$

N. Min. $\left(\mathrm{mg} \mathrm{kg}^{-1}\right) \quad$ Max. $\left(\mathrm{mg} \mathrm{kg}^{-1}\right)$ Mean $\left(\mathrm{mg} \mathrm{kg}^{-1}\right) \quad$ Range

$N \quad$ Min. $\left(\mathrm{mg} \mathrm{kg}^{-1}\right) \quad$ Max. $\left(\mathrm{mg} \mathrm{kg}^{-1}\right) \quad$ Mean $\left(\mathrm{mg} \mathrm{kg}^{-1}\right)$ 


\section{ELSEVIER_ENPO_7234}

\begin{tabular}{|c|c|c|c|c|c|c|c|c|c|c|c|c|c|c|c|c|}
\hline Fluorene & 0.02 & 43 & $<0.02$ & 4.80 & 0.73 & 4.80 & 58 & $<0.02$ & 2.70 & 0.45 & 2.70 & 36 & $<0.02$ & 6.25 & 0.88 & 6.25 \\
\hline Phenanthrene & 0.02 & 43 & $<0.02$ & 3.64 & 0.47 & 3.64 & 58 & 0.02 & 3.15 & 0.81 & 3.13 & 36 & $<0.02$ & 6.86 & 0.76 & 6.86 \\
\hline Anthracene & 0.02 & 43 & $<0.02$ & 4.22 & 0.41 & 4.22 & 58 & $<0.02$ & 2.57 & 0.36 & 2.57 & 36 & $<0.02$ & 6.86 & 0.75 & 6.86 \\
\hline Pyrene & 0.02 & 43 & $<0.02$ & 1.03 & 0.30 & 1.03 & 58 & $<0.02$ & 2.83 & 0.56 & 2.83 & 36 & $<0.02$ & 1.73 & 0.52 & 1.73 \\
\hline Benz[a]anthracene & 0.02 & 43 & $<0.02$ & 0.88 & 0.18 & 0.88 & 58 & $<0.02$ & 2.61 & 0.39 & 2.61 & 36 & $<0.02$ & 1.01 & 0.15 & 1.01 \\
\hline Chrysene & 0.02 & 43 & $<0.02$ & 1.17 & 0.18 & 1.17 & 58 & $<0.02$ & 1.07 & 0.22 & 1.07 & 36 & $<0.02$ & 0.55 & 0.09 & 0.55 \\
\hline Benz[b]fluoranthene & 0.02 & 43 & $<0.02$ & 0.15 & 0.03 & 0.15 & 58 & $<0.02$ & 1.33 & 0.23 & 1.33 & 36 & $<0.02$ & 0.18 & 0.04 & 0.18 \\
\hline Benzo[k]fluoranthene & 0.02 & 43 & $<0.02$ & 0.15 & 0.03 & 0.15 & 58 & $<0.02$ & 1.23 & 0.19 & 1.23 & 36 & $<0.02$ & 0.18 & 0.04 & 0.18 \\
\hline Benzo[a]pyrene & 0.02 & 43 & $<0.02$ & 0.73 & 0.17 & 0.73 & 58 & $<0.02$ & 1.80 & 0.14 & 1.80 & 36 & $<0.02$ & 9.36 & 0.68 & 9.36 \\
\hline Indeno[1,2,3-cd]pyrene & 0.02 & 43 & $<0.02$ & 0.22 & 0.02 & 0.22 & 58 & $<0.02$ & 1.48 & 0.23 & 1.48 & 36 & $<0.02$ & 0.82 & 0.06 & 0.82 \\
\hline Dibenzo[a,h]anthracene & 0.02 & 43 & $<0.02$ & 0.15 & 0.03 & 0.15 & 58 & $<0.02$ & 1.82 & 0.22 & 1.82 & 36 & $<0.02$ & $<0.02$ & - & - \\
\hline Benzo[g,h,i]perylene & 0.02 & 43 & $<0.02$ & 0.22 & 0.02 & 0.22 & 58 & $<0.02$ & 1.48 & 0.22 & 1.48 & 36 & $<0.02$ & 0.33 & 0.02 & 0.33 \\
\hline
\end{tabular}

LOQ, Limit of quantitation. It is the lowest concentration at which an analyte can be reliably detected (Mitra, 2003).

$N$, Number of samples.

PAH, Polycyclic aromatic hydrocarbons.

Percent recoveries of spiked surrogates for the three sites, as shown in Table 2, are within the acceptable range of 40-120-\% (USEPA, 1999), which is typical of a reasonably accurate PAH extraction procedure.

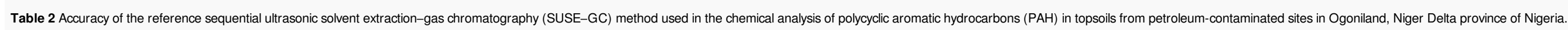

\begin{tabular}{|c|c|c|c|c|c|}
\hline \multirow[t]{2}{*}{ Sampling site } & \multirow[t]{2}{*}{ No. of samples } & \multirow[t]{2}{*}{ Spiked $\left(\mathrm{mg} \mathrm{kg}^{-1}\right)$} & \multicolumn{2}{|c|}{ Measured $\left(\mathrm{mg} \mathrm{kg}^{-1}\right)^{\mathrm{a}}$} & \multirow[t]{2}{*}{$\%$ Recovery of spiked surrogates } \\
\hline & & & 2-Fluorobiphenyl & o-Terphenyl & \\
\hline Baraboo & 43 & 2.50 & 1.36 & 1.33 & 53-54 \\
\hline Bomu 1 & 58 & 2.50 & 1.94 & 1.63 & $65-78$ \\
\hline Bomu 2 & 36 & 2.50 & 1.19 & 1.40 & $48-56$ \\
\hline
\end{tabular}

a Mean concentrations of the spiked surrogates.

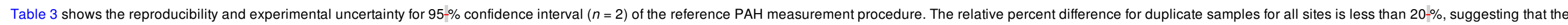

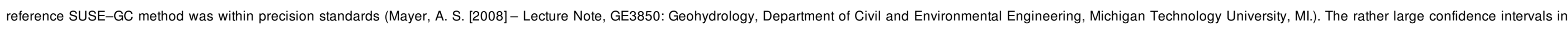

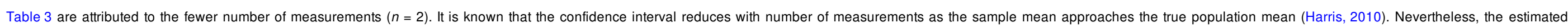
confidence interval suggests with $95-\%$ confidence that the true mean $\mathrm{PAH}$ value from the duplicate measurements would lie within the estimated range.

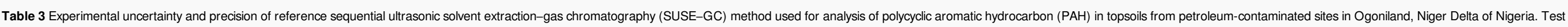
sample was randomly chosen for each site.

\begin{tabular}{|c|c|c|c|c|}
\hline \multirow[t]{2}{*}{ Sampling site } & \multirow[t]{2}{*}{ First duplicate $\left(\mathrm{mg} \mathrm{kg}^{-1}\right)$} & \multirow[t]{2}{*}{ Second duplicate $\left(\mathrm{mg} \mathrm{kg}^{-1}\right)$} & \multirow[t]{2}{*}{ Relative percent difference $(\%)$} & Confidence interval \\
\hline & & & & Mean \pm uncertainty ${ }^{\mathrm{a}}\left(\mathrm{mg} \mathrm{kg}^{-1}\right)$ \\
\hline Baraboo & 3.67 & 3.22 & 13 & $3.45 \pm 2.86$ \\
\hline Bomu 1 & 4.49 & 3.88 & 14 & $4.18 \pm 3.84$ \\
\hline Bomu 2 & 2.89 & 2.66 & 8 & $2.78 \pm 1.42$ \\
\hline
\end{tabular}




\section{ELSEVIER_ENPO_7234}

${ }^{a}$ Experimental uncertainty for $95-\%$ confidence interval for duplicate measurements.

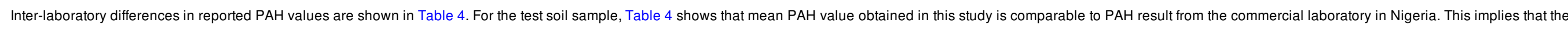

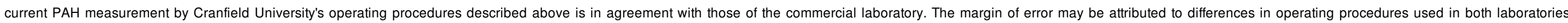
(Table 4). Moreover, inter-laboratory differences in reported values of an analyte in chemical analysis are a common occurrence (Risdon et al., 2008).

Table 4 Inter-laboratory differences in reported PAH values and selected rubrics in operating procedures for test soil sample from petroleum-contaminated site in Baraboo in Ogoniland, Niger Delta of Nigeria.

\begin{tabular}{|c|c|c|}
\hline Analyte and selected rubrics & This study & Commercial laboratory \\
\hline PAH $\left(\mathrm{mg} \mathrm{kg}^{-1}\right)$ & $3.45 \pm 2.86$ & $2.73^{\mathrm{a}}$ \\
\hline Percent error $(\%)^{b}$ & 26 & - \\
\hline Quantitation method & Internal standard & External standard \\
\hline Extraction method & Sequential ultrasonic solvent extraction & Sonication water bath (5-hout sonication) \\
\hline Extracting solvent(s) & Dichloromethane and hexane (1:1) & $n$-Pentane \\
\hline Surrogate standard(s) & 2-Fluorobiphenyl and o-Terphenyl & 1-Chlorooctadecane \\
\hline
\end{tabular}

a Uncertainty was not reported.

${ }^{\mathrm{b}}$ Commercial laboratory PAH result was taken as the "known" value.

\subsection{The PAH partial least-squares regression models}

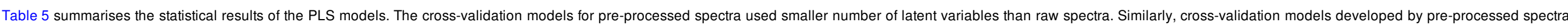

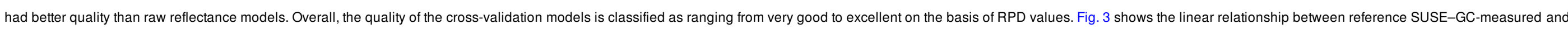

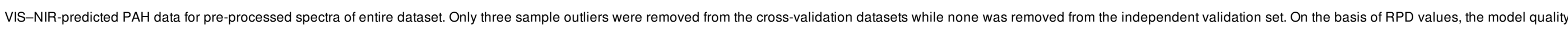

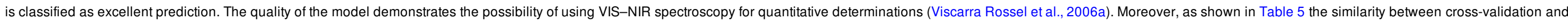

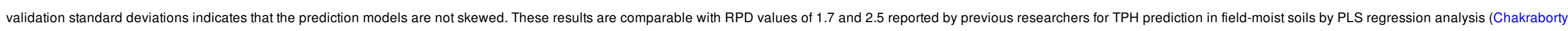
et al., 2010, 2012).

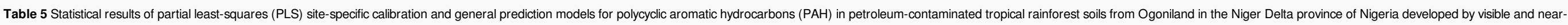
infrared (VIS-NIR) spectroscopy.

\begin{tabular}{|c|c|c|c|c|c|c|c|c|c|c|c|}
\hline \multirow[t]{2}{*}{ Sampling site } & \multirow[t]{2}{*}{ No. of samples } & \multicolumn{5}{|c|}{ Reflectance spectra } & \multicolumn{5}{|c|}{ Combined pre-processing spectra ${ }^{a}$} \\
\hline & & $r^{2}$ & $\operatorname{RMSE}\left(\mathrm{mg} \mathrm{kg}^{-1}\right)$ & SD & RPD & LV & $r^{2}$ & RMSE $\left(\mathrm{mg} \mathrm{kg}^{-1}\right)$ & SD & RPD & LV \\
\hline \multicolumn{12}{|l|}{ Cross-validation set } \\
\hline Baraboo & 43 & 0.78 & 0.82 & 1.81 & 2.20 & 8 & 0.84 & 0.64 & 1.81 & 2.81 & 6 \\
\hline Bomu 1 & 58 & 0.76 & 1.41 & 2.95 & 2.09 & 11 & 0.83 & 1.22 & 2.95 & 2.41 & 3 \\
\hline Bomu 2 & 36 & 0.77 & 1.98 & 4.08 & 2.07 & 5 & 0.83 & 1.42 & 4.08 & 2.87 & 7 \\
\hline General & 107 & 0.69 & 1.61 & 3.05 & 1.81 & 11 & 0.82 & 1.30 & 3.05 & 2.34 & 8 \\
\hline \multicolumn{12}{|l|}{ Validation set } \\
\hline General & 30 & 0.77 & 1.95 & 3.63 & 1.86 & 11 & 0.89 & 1.16 & 3.63 & 3.12 & 8 \\
\hline
\end{tabular}

LV, Latent variable. 


\section{ELSEVIER_ENPO_7234}

RMSE, Root-mean-square error.

RPD, Ratio of prediction deviation.

SD, Standard deviation.

${ }^{a}$ Combination of maximum normalisation, and first derivative and smoothing by Savitzky-Golay.

Cross-validation (78\%) set

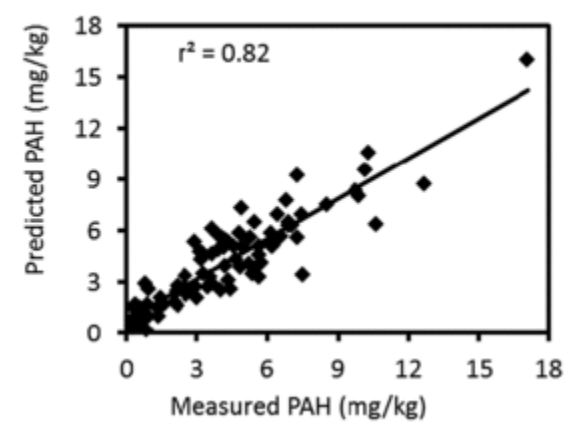

Validation (22\%) set

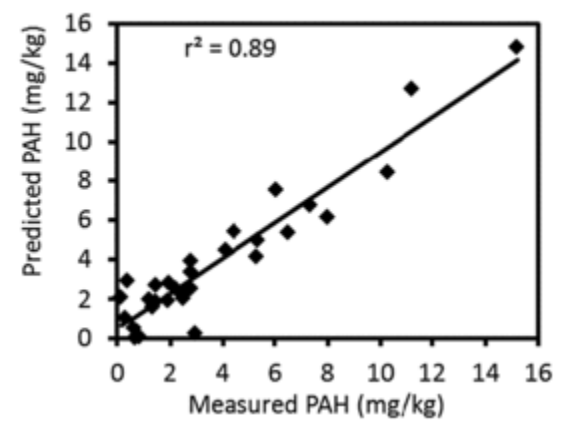

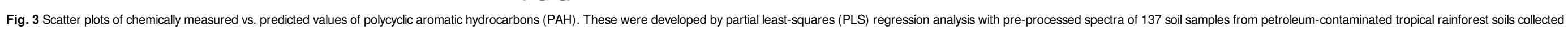
from Ogoniland, Niger Delta, Nigeria.

\subsection{Spectral reflectance of petroleum-contaminated tropical rainforest soils}

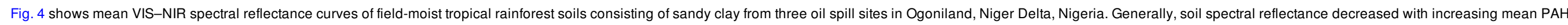

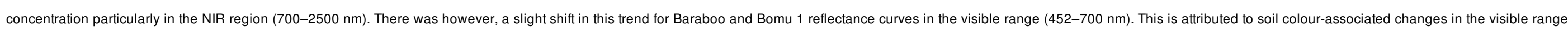

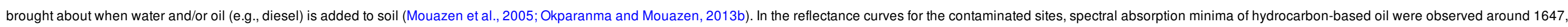

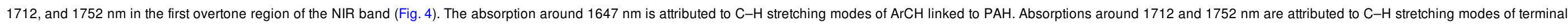

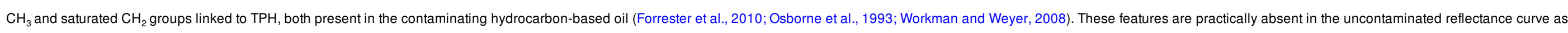
shown by the number of reflectance shoulders that appeared in the reflectance curves between 1452 and $1952 \mathrm{~nm}$ (Fig. 4). 


\section{ELSEVIER_ENPO_7234}

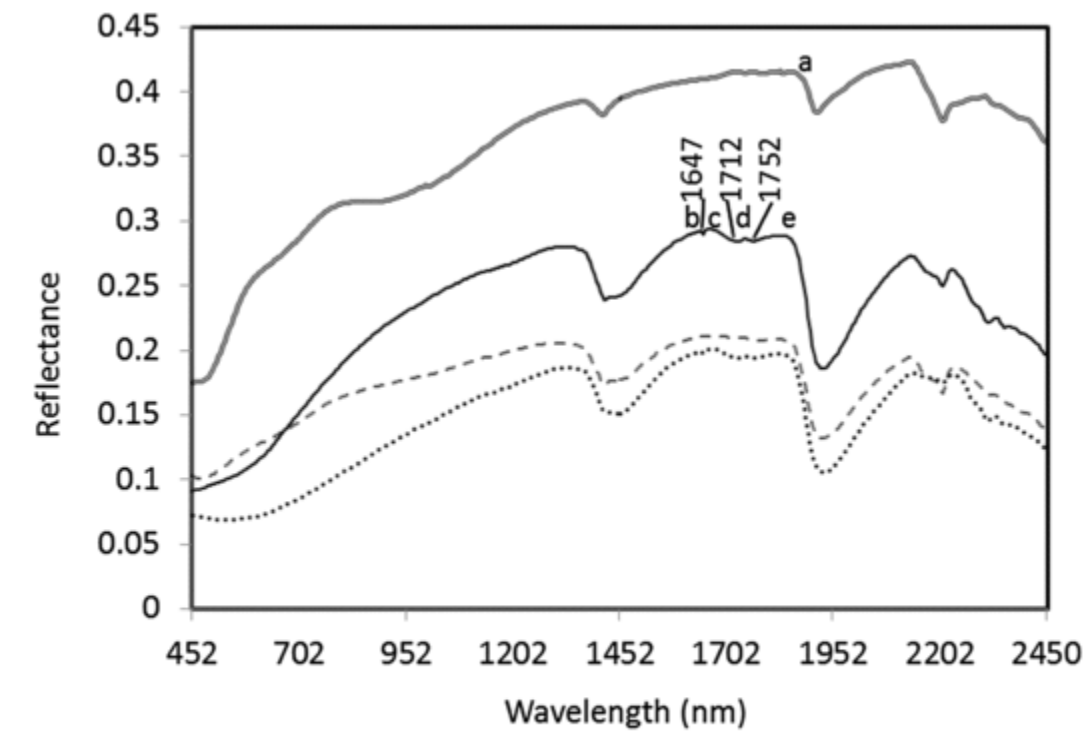

\section{........ Bomu 2 (5.39 mgkg-1 PAH) - - - Bomu 1 (4.47 mgkg-1 PAH) \\ —Baraboo (3.38 mgkg-1 PAH) Uncontaminated}

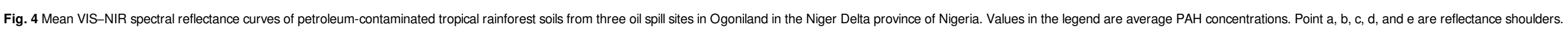

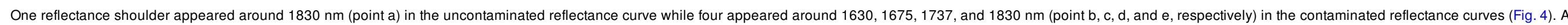

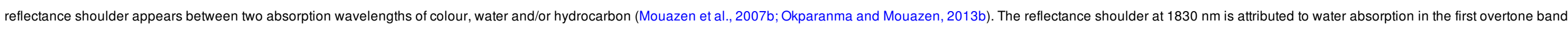

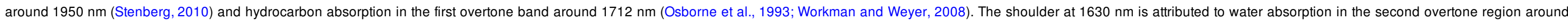

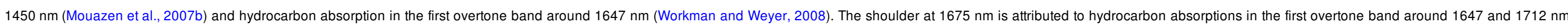

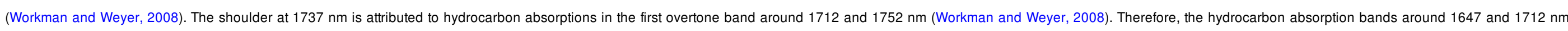
differentiate the uncontaminated from contaminated reflectance curves (Fig. 4).

\subsection{Regression coefficients}

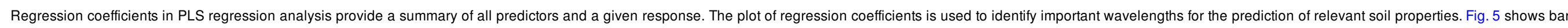

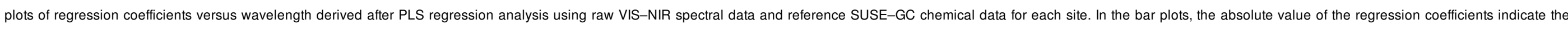

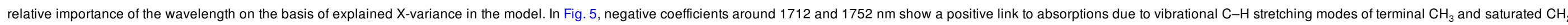

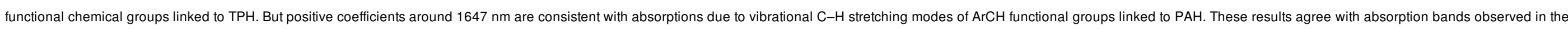

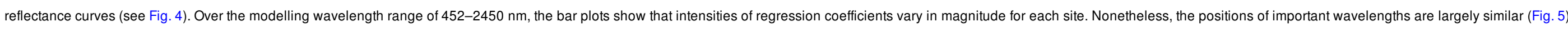

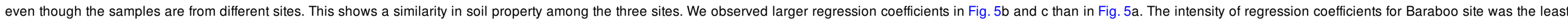

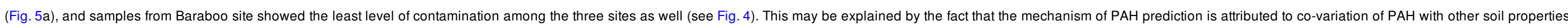

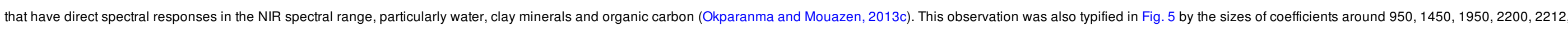

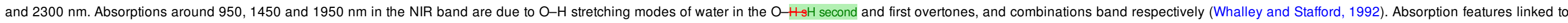

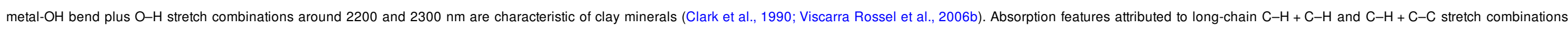




\section{ELSEVIER_ENPO_7234}

around $2150 \mathrm{~nm}$ and $2212 \mathrm{~nm}$ are unique to soil organic matter (Forrester et al., 2010).
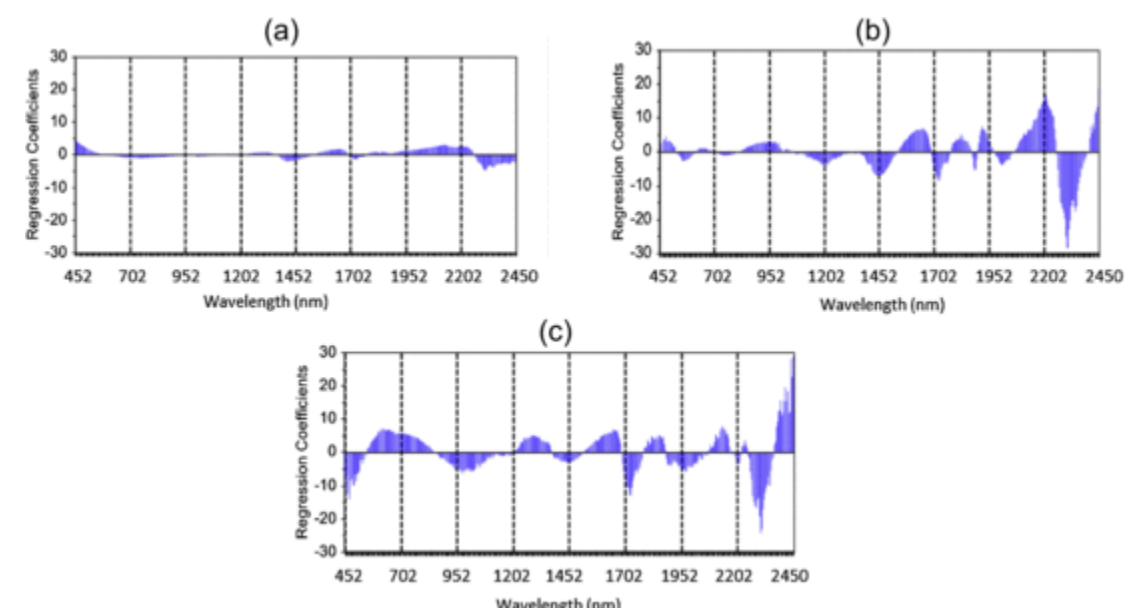

Online view $\square$ Print view

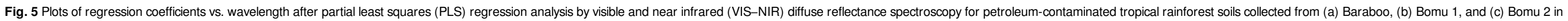
Ogoniland, Niger Delta, Nigeria.

\section{Conclusions}

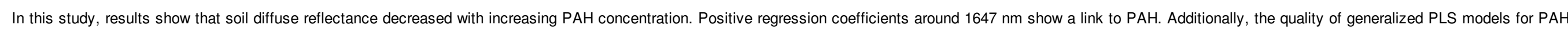

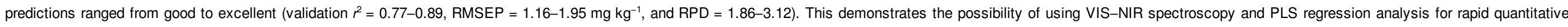

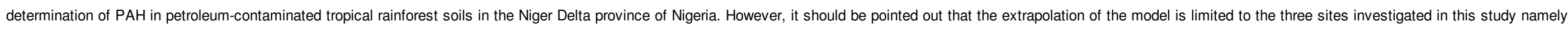

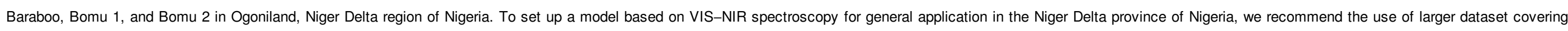
both the concentration range and all the other sources of variability in oil spill sites in the Niger Delta region.

\section{Acknowledgements}

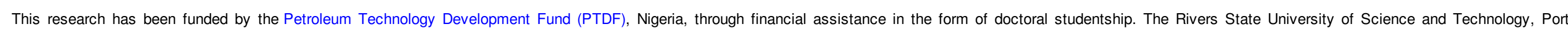
Harcourt, Nigeria, also provided support through its Academic Staff Development Program.

\section{References}

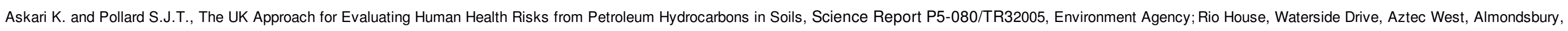
Bristol, UK, 1-22.

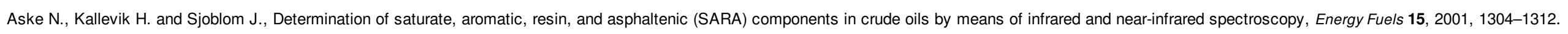

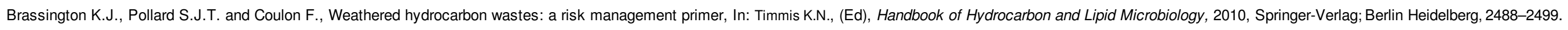

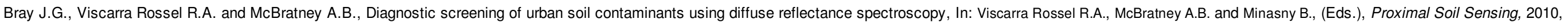
Springer-Verlag; Berlin Heidelberg, 191-199.

CAMO Software, The Unscrambler ${ }^{\circledR}$ X Version 10.2 User's Guide, 2012, CAMO Software AS; Nedre Vollgate, Oslo, Norway. 


\section{ELSEVIER_ENPO_7234}

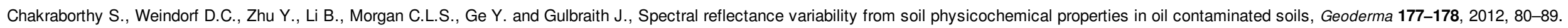

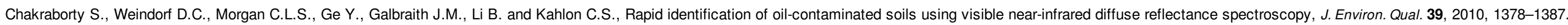
Clark R.N., King T.V.V., Klejwa M., Swayze G. and Vergo N., High spectral resolution reflectance spectroscopy of minerals, J. Geophys. Res. 95, 1990, 12653-12680.

Department of Petroleum Resources, Environmental Guidelines and Standards for the Petroleum Industry in Nigeria (EGASPIN), 2002, Ministry of Petroleum and Natural Resources; Abuja, Nigeria, 314.

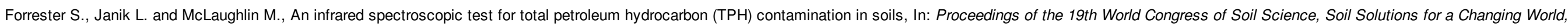

Brisbane, Australia, August 1-6, 2010, 13-16.

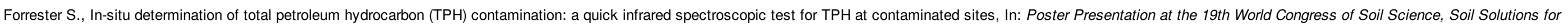

a Changing World, Brisbane, Australia, August 1-6, 2010.

Forrester, S.F., Janik, L.J., McLaughlin, M.J., 2011. Method of Contaminant Prediction. PCT Patent WO/2011/035391. Date issued: 31 March.

Fuller M.P. and Griffiths P.R., Diffuse reflectance measurements by infrared Fourier transform spectrometry, Anal. Chem. 50 (13), 1978, $1906-1910$.

Graham K.N., Evaluation of Analytical Methodologies for Diesel Fuel Contaminants in Soil, (M.Sc. thesis)1998, The University of Manitoba; Canada, (Unpublished results).

Harris D.C., Quantitative Chemical Analysis, eighth ed., 2010, W.H. Freeman and Company; New York.

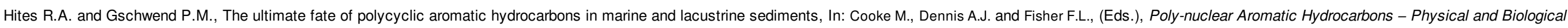

Chemistry, 1982, Bettelle Press; Columbus, Ohio, USA, 357-365.

Kuang B. and Mouazen A.M., Effect of spiking strategy and ratio on calibration of on-line visible and near infrared soil sensor for measurement in European farms, Soil Till. Res. 128, 2013, 125-136.

Maleki M.R., Mouazen A.M., Ramon H. and De Baerdemaeker J., Optimization of soil VIS-NIR sensor-based variable rate application system of soil phosphorus, Soil Till. Res. 94, 2007 , 239-250.

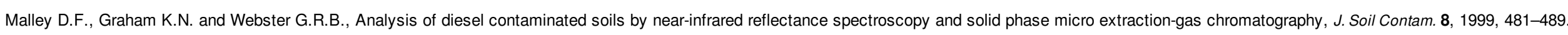

Mitra S., Sample Preparation Techniques in Analytical Chemistry, 2003, Wiley and Sons, Inc., Publication; Hoboken, NJ, USA.

Mouazen A.M., De Baerdemaeker J. and Ramon H., Towards development of on-line soil moisture content sensor using a fibre-type NIR spectrophotometer, Soil Till. Res. 80, 2005, 171-183.

Mouazen A.M., Maleki M.R., De Baerdemaeker J. and Ramon H., On-line measurement of some selected soil properties using a VIS-NIR sensor, Soil Till. Res. 93 (1), 2007 a, 13-27.

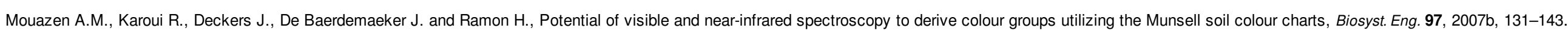

Naes T., Isaksson T., Fearn T. and Davies T., A User Friendly Guide to Multivariate Calibration and Classification, 2002, NIR Publications; Chichester, UK.

Niger Delta Environmental Survey, Background and Mission: Briefing Note 1, 1995, Publication of the Steering Committee, NDES; Falomo, Lagos, Nigeria, 1-7.

Okoro D. and Ikolo A.O., Sources and compositional distribution of polycyclic aromatic hydrocarbons in soils of Western Niger Delta, J. Appl. Sci. Technol. 12 (1-2), $2007,35-40$.

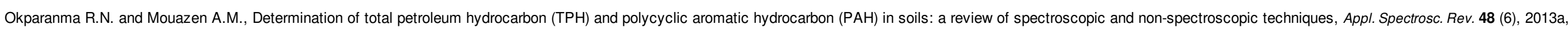
458-486.

Okparanma R.N. and Mouazen A.M., Combined effects of oil concentration, clay and moisture contents on diffuse reflectance spectra of diesel-contaminated soils, Water Air Soil Pollut. 224 (5), $2013 \mathrm{~b}, 1539-1556$.

Okparanma R.N. and Mouazen A.M., Visible and near-infrared spectroscopy analysis of a polycyclic aromatic hydrocarbon in soils, ScientificWorldJournal 2013c, (Article in reviewsubmitted for publieation). 


\section{ELSEVIER_ENPO_7234}

Osborne B.G., Fearn T. and Hindle P.H., Practical NIR Spectroscopy - with Applications in Food and Beverage Analysis, second ed., 1993, Longman Group UK Limited; England.

Patnaik P., Handbook of Environmental Analysis: Chemical Pollutants in Air, Water, Soil and Solid Wastes, 1997, CRC Press Inc.; Boca Raton, FL.

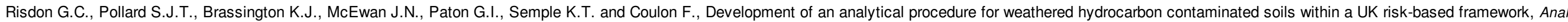
Chem. 80 (18), 2008, 7090-7096.

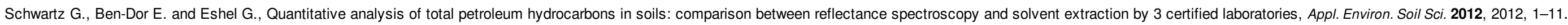

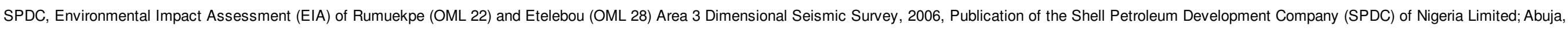

Nigeria, Available: http://www.shell.com.ng/environment-society/environment-impact-assessments.html, (accessed 25.02.13.).

Stenberg B., Effects of soil sample pretreatments and standardized rewetting as interacted with sand classes on VIS-NIR predictions of clay and soil organic carbon, Geoderma 158, 2010, 15-22.

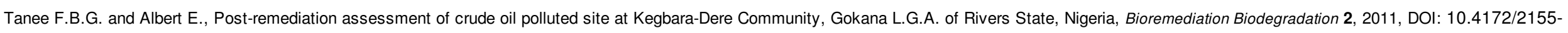

6199.1000122, (accessed 26.02.13.).

UNEP, Environmental Assessment of Ogoniland, 2011, United Nations Environment Programme (UNEP); Nairobi, Kenya, Available: http://www.unep.org, (accessed 29.10.12.).

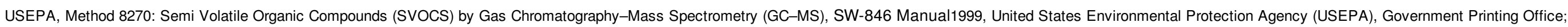
Washington, DC, USA.

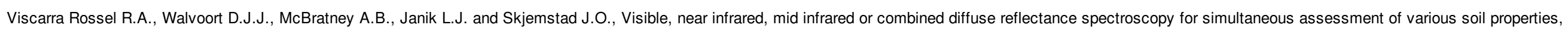

Geoderma 131, 2006a, 59-75.

Viscarra Rossel R.A., McGlynn R.N. and McBratney A.B., Determining the composition of mineral-organic mixes using UV-vis-NIR diffuse reflectance spectroscopy, Geoderma 137, $2006 \mathrm{~b}, 70-82$.

Wang Z. and Fingas M., Differentiation of the sources of spilled oil and monitoring of the oil weathering process using gas chromatography-mass spectroscopy, J. Chromatogr. A 712, 1995, 321-343.

Whalley W.R. and Stafford J.V., Real-time sensing of soil water content from mobile machinery: options for sensor design, Comput. Electron. Agr. 7, 1992, 269-358.

Willey R.R., Fourier transform infrared spectrophotometer for transmittance and diffuse reflectance measurements, Appl. Spectrosc. 30 (6), $1976,593-601$.

Williams P.C. and Sobering D.C., Attempts at standardization of hardness testing of wheat. II. The near-infrared reflectance method, Cereal Foods World 31, 1986, 417-420.

Workman J., Jr. and Weyer L., , Practical Guide to Interpretive Near-infrared Spectroscopy, 2008, CRC Press, Taylor and Francis Group; Boca Raton, FL, USA.

Yunker M.B. and MacDonald R.W., Composition and origins of polycyclic aromatic hydrocarbons in the Mackenzie River and on the Beaufort Sea Shelf, J. Arct. Inst. N. Am. 48 (2), $1995,118-129$.

\section{Highlights}

- We model NIR diffuse reflectance spectra for PAH prediction in contaminated soils.

- Soil diffuse reflectance decreases with increasing PAH concentration.

- Mechanism of prediction relies on co-variation of PAH with other soil properties.

- Positions of important wavelengths are largely similar for studied sites.

- Positive regression coefficients around $1647 \mathrm{~nm}$ show a link to PAH. 


\section{ELSEVIER_ENPO_7234}

\section{Queries and Answers}

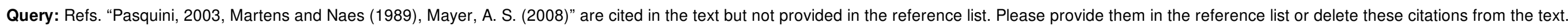
Answer:

Pasquini, C., Near infrared spectroscopy: fundamentals, practical aspects and analytical applications. J. Braz. Chem. Soc., 14(2), 2003, 198-219.

Martens, H. and Naes, T., Multivariate Calibration, second ed., 1989, John Wiley and Sons; Chichester, UK.

Mayer, A.S., Lecture Note, GE3850: Geohydrology, Department of Civil and Environmental Engineering, Michigan Technology University, MI, 2008, Unpublished data.

Query: Please update Okparanma and Mouazen, submitted for publication.

Answer: Article is in review.

Query: Please confirm that given names and surnames have been identified correctly.

Answer: Confirmed ok 